\title{
Self-administered physical exercise training as treatment of neck and shoulder pain among military helicopter pilots and crew: a randomized controlled trial
}

\author{
Mike Murray ${ }^{1 *}$, Britt Lange ${ }^{2}$, Bo Riebeling Nørnberg ${ }^{3}$, Karen Søgaard ${ }^{1}$ and Gisela Sjøgaard ${ }^{1}$ (D)
}

\begin{abstract}
Background: Neck pain is frequent among military helicopter pilots and crew-members, and pain may influence individual health and work performance. The aim of this study was to examine if an exercise intervention could reduce neck pain among helicopter pilots and crew-members.
\end{abstract}

Methods: Thirty-one pilots and thirty-eight crew-members were randomized to either an exercise-training-group $(n=35)$ or a reference-group $(n=34)$. The exercise-training-group received 20-weeks of specific neck/shoulder training. The reference-group received no training. Primary outcome: Intensity of neck pain previous 3-months (scale 0-10). Secondary outcomes: additional neck/shoulder pain intensity variables and pressure-pain-threshold in the trapezius muscle (TRA) and upper-neck-extensor muscles (UNE). Regular training adherence was defined as $\geq 1$ training session a week. Statistical analyses performed were intention-to-treat and per-protocol. Students $t$-test was performed $(p<0.05)$.

Results: Intensity of neck pain previous 3-months at baseline was: $2.2 \pm 1.8$ and previous 7 -days: $1.0 \pm 1.5$, and pressure-pain-threshold in TRA and UNE (right/left) was in kPa: $424 \pm 187 / 434 \pm 188$ and $345 \pm 157 / 371 \pm 170$ in the exercise-training-group, and $416 \pm 177$ / $405 \pm 163$ and $334 \pm 147$ / $335 \pm 163$ in the reference-group, with no differences between groups. Intention-to-treat-analysis revealed no significant between-group-differences in neck pain intensity and pressure-pain-threshold. Between-group-differences, including participants who trained regularly $(n=10)$ were also non-significant. Within-group-changes were significant among participants with regular training adherence in the exercise-training-group regarding intensity of neck pain previous 3-months (from $2.2 \pm 0.6$ to $1.3 \pm 1.3, p=0.019$ ). Likewise, within the whole exercise-training-group, neck pain previous 7-days decreased (from $1.0 \pm 1.4$ to $0.6 \pm 1.1, p=0.024)$. Additional within-group-changes regarding pressure-pain-threshold in $\mathrm{kPa}$ were for the reference-group a reduction in TRA and UNE (right/left) to: $342 \pm 143$ / $332 \pm 154$ and $295 \pm 116 / 292 \pm 121$ implying increased pain sensitivity, while for the exercise-training-group only a reduction in left TRA was seen: $311 \pm 113$.

Conclusions: The exercise intervention did not reduce neck pain among helicopter pilots and crew-members as no significant between-group-differences were found. However, some trends were demonstrated as some neck pain intensity and sensitivity improved more within the exercise-training-group but not within the reference-group. The lack of effect may be due to low adherence since only $~ 1 / 3$ of subjects in the exercise-training-group engaged in regular training which may be due to the self-administration of the training.

(Continued on next page)

\footnotetext{
* Correspondence: mike_ster@hotmail.com

1 Department of Sports Science and Clinical Biomechanics, University of

Southern Denmark, Odense, Denmark

Full list of author information is available at the end of the article
} 
(Continued from previous page)

Trial registrations: Ethical committee of Southern Denmark (S-20120121) 29 August, 2012. Clinical Trail Registration (NCT01926262) 16 August, 2013.

Keywords: Musculoskeletal disorders, Neck, Pain, Exercise, Pilot, Crew-member, Clinical trial, Intervention, Pressure-pain-threshold

\section{Background}

Neck pain among helicopter pilots and crew-members is a challenging problem within modern air forces [1]. The 3months prevalence of neck pain among helicopter pilots has previously been estimated to $57 \%$ with $32 \%$ of pilots reporting recurrent pain episodes [2]. These prevalence rates are high compared to a 6 -months prevalence of $\sim$ $30 \%$ and a 1 -year prevalence of $\sim 40 \%$ within the general working population [3]. Neck pain represents an individual health concern at leisure and may as well influence pilots and crew-members level of concentration [4], thereby potentially affecting operational safety. Neck pain may also impact operational capacity within air forces through increased sickness leave [1]. Limited research has been conducted on neck pain among helicopter pilots and especially crew-members. With some exceptions, due to work tasks [5], helicopter pilots and crew-members undergo similar in-flight exposures such as the use of a flight helmet and helmet mounted devices like night vision goggles (NVG). Especially loading of the cervical spine has been reported as a risk factor for neck pain and discomfort in pilots and crew-members [6]. Loading of the cervical spine has also been measured in laboratory settings combining different head positions [7, 8], and during real flight [9]. Based on current results loading of the cervical spine poses a heavy burden on the cervical spine musculature of pilots as well as crew-members which may potentially result in neck pain episodes. It is therefore imperative that both groups are addressed regarding flight related neck pain. Physical exercise training has been found effective as a deterrent against neck pain in a number of large intervention studies conducted within other working populations [10-12]. Specifically tailored exercise interventions have proven particularly effective [13, 14]. Few exercise interventions have been conducted among helicopter pilots and crew-members with successful outcome regarding reduction of neck pain. Theoretically, exercise training may increase individual capacity and reduce the relative workload on the cervical musculature, thereby reducing the risk of developing neck pain [15]. However, no currently evidence-based guidelines, contributing to the prevention and clinical management of neck pain among helicopter pilots and crew, exist within the Royal Danish Air Force (RDAF). It is therefore important that further exercise studies are conducted in order to increase available knowledge.
The aims of this study were to 1 ) determine the prevalence of neck and shoulder pain among military helicopter pilots and crew-members within the RDAF, and to 2) evaluate the effectiveness of a 20-week exercise intervention on intensity of neck pain among helicopter pilots and crew-members. The hypothesis tested was that the exercise intervention resulted in a reduction in self-reported intensity of neck pain previous 3-months. Secondarily, it was tested if objectively measured pain sensitivity in the neck and shoulder muscles as well as other neck/shoulder pain intensity variables were reduced.

\section{Methods \\ Design of the study}

The study design was a parallel group, single blinded, randomized, controlled trial. The intervention period was 20weeks with pre-intervention baseline measurements and post-intervention follow-up measurements. A detailed protocol paper has been published previously [13]. The trial was conducted within the RDAF from November 2013 to April 2014. All participants volunteered and gave their written informed consent before participation. The trial was approved by the local Ethics Committee of Southern Denmark (S-20120121) and qualified for registration in ClinicalTrials.gov (NCT01926262).

\section{Participants and randomization}

Fifty military helicopter pilots and fifty-eight crew-members from two different RDAF squadrons were invited to participate in the study. Participants were informed about the project at briefings, by email, and by telephone. Thirty one pilots (2 female and 29 male) and thirty-eight crewmembers (male) agreed to participate. Inclusion criteria comprised: 1) profession as a helicopter pilot or crewmember (technician, systems operator, tactical helicopter observer and/or navigator), 2) maintaining operational flight status at enrollment, 3) operational flying within the previous 6-months. Exclusion criteria comprised: 1) participation in a training intervention during the previous 12months. Participants flow is depicted in Fig. 1. Participants were assigned a random identification number at enrollment by an authorized person with no relation to the study. After pre-intervention assessments participants were randomized 1:1 to either an exercise-training-group (ETG) or to a reference-group (REF). Participants were stratified according to the following nested criteria to ensure 


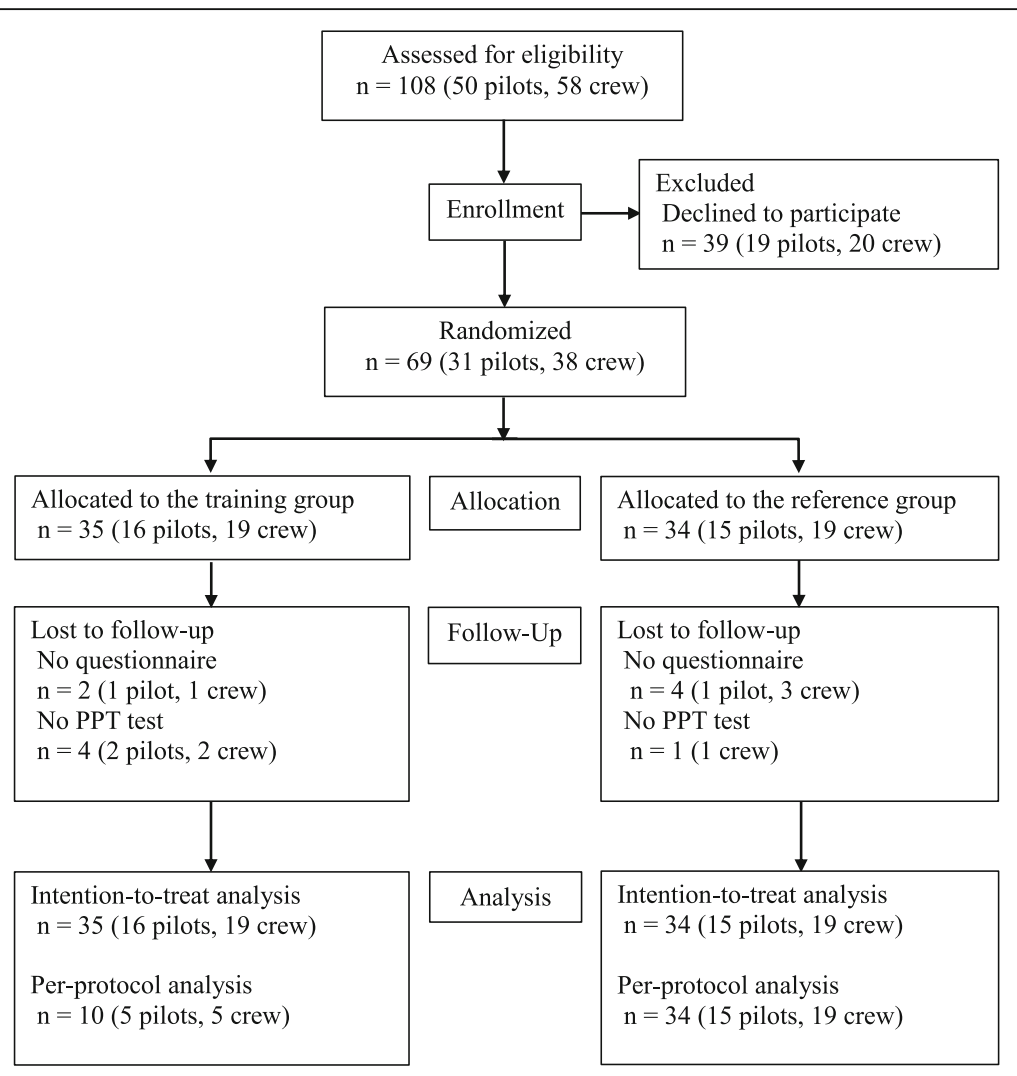

Fig. 1 Flow diagram

comparability between the ETG and REF: 1) squadron (722 squadron or 724 squadron), 2) profession (pilot or crewmember), 3 ) age ( $<$ or $\geq 40$ years of age), and 4 ) flying experience ( $<$ or $\geq 2500 \mathrm{~h}$ ). The random identification numbers within each stratum were drawn from an opaque, tossed bag. Alternately, the first number in the first strata was allocated to either the ETG or REF depending on the flip of a coin. The first number in the second strata was allocated to the opposite group, compared to the last number in the previous strata, and so forth. The randomization procedure was carried out by a blinded custodian (last author) using the random identification numbers assigned to the participants. Data analysts and statistician were blinded to the random group allocation of the participants.

\section{Measurements}

All methods have been previously described [13], and will therefore only be explained briefly. Anthropometric measurements included: height, seated height, weight and body mass index (BMI) (Composition Analyzer Tanita Corporation of America, USA).

Participants' pressure-pain-threshold (PPT) was measured in the neck and shoulder muscles. PPT was measured bilaterally for the trapezius muscle (TRA) (20\% medially to half the distance between the lateral edge of the acromion and seventh cervical vertebra) [16], the upper neck muscles (UNE) $(2 \mathrm{~cm}$ laterally to the vertical line of the axis in level with the 4'th cervical vertebra) [17, 18], and the anterior tibialis muscle (TIA) (as the point of reference) [19]. A handheld electronic pressure algometer was used (Type II Algometer, Somedic Production AB, Sweden). The algometer was pistol-shaped with a pressure-sensitive strain gauge at the tip. The contact area had a diameter of $1 \mathrm{~cm}^{2}$. Compression pressure was applied perpendicularly to the skin with a rate of $20 \mathrm{kPa} / \mathrm{s}$. A digital display on the pressure algometer was used to keep the rate of pressure stable. Measurements were performed three times in a fixed order: 1) right TRA, 2) left TRA, 3) right UNE, 4) left UNE, and 5) right TIA. A rest period of approximately $1 \mathrm{~min}$ was given between measurements conducted on the same PPT point. Participants were given a hand held control switch and were instructed to immediately press the switch when the sensation of "pressure" changed to "pain". When the switch was pushed the compression was stopped and the pressure was released [20]. A low level of pain sensitivity therefore equals a high PPT value, and a high level of pain sensitivity equals a low PPT value. The maximum applied pressure registered was recorded before resetting the algometer. A maximal pressure of $1000 \mathrm{kPa}$ was allowed for TRA and TIA and $700 \mathrm{kPa}$ for the UNE. The algometer was calibrated before each test. 
Measurements were conducted by an experienced sports scientist.

\section{Questionnaire}

An online based questionnaire was applied to participants pre- and post-intervention. The questionnaire was confidential using the assigned identification numbers. A modified version of the validated Nordic Musculoskeletal Questionnaire [21] was used to assess the prevalence and intensity of musculoskeletal symptoms in the following body regions: neck and shoulders, upper back, elbows, low back, wrists/hands, hips/thighs, knees, and ankles/feet according to: 1) the number of days with pain or complaints in (body region) the previous 12-months (possible answers: 0 days, $1-7$ days, $8-30$ days, 31 90 days, $>90$ days, or every day), 2) inability to perform daily working tasks due to complaints in (body region) the previous 3-months (possible answers: yes or no), 3) intensity of (body region) pain previous 3-months was assessed on a scale from 1-10 (10 = worst possible pain imaginable $)$ and to this was added $0=$ no pain, resulting overall in an 11 point numeric box scale, and 4) intensity of (body region) pain previous 7-days depicted on the same box scale. All questions were accompanied by chart illustrations of the body region in focus. Furthermore, participants were inquired about the amount of total flying hours in fixedwing aircraft, rotary-wing aircraft, and flying hours with NVG. Lastly, the questionnaire included a number of health and work related questions [13].

Participants randomized to the ETG received an additional questionnaire regarding: 1) their motivation to train, 2) expectations, 3) training adherence, and 4) adverse training effects. Training adherence was measured by inquiring: "You were instructed to train $3 \times 20 \mathrm{~min}$ a week. How did you succeed?" Possible answers: "I trained regularly 2-3 times a week, I trained regularly 12 times a week, I trained irregularly, but at least 4 times a month (approximately once a week), I trained irregularly but at least 2-3 times a month, I trained some, but stopped training after a while, and I did not use the training offer".

\section{Exercise intervention}

Participants in the ETG received 20-weeks of strength, endurance, and coordination training targeting the neck and shoulder muscles. Training was divided into training sessions of $3 \times 20$ min per week. The training programme was evidence based and designed by an interdisciplinary team of sports exercise training specialists, physiotherapists, doctors and chiropractors. The training programme was composed of ten training exercises divided into three categories: 1) two warming up exercises, 2) six neck exercises, and 3) two shoulder exercises. All exercises have been described previously [13] and training videos are available online [22].

Every training session was initiated with one or two warming up exercises recruiting the deep cervical muscle groups. Exercises included: cervical flexion from a supine position and cervical rotation from an erect position. The warming up exercises were performed using 3 sets of 15 repetitions. Intensity was increased as participants progressed. These two exercises also aimed to warm up the neck before engaging in more strenuous training exercises. The warming up exercises were followed by training exercises for the larger neck muscles including: cervical extension, cervical flexion (straight forward and oblique angels), and lateral flexion. Finally, participants performed two exercises for the shoulder girdle including: shrugs and reverse flies. Neck and shoulder exercises were performed using elastic training bands for resistance (Thera-Band ${ }^{\circ}$, The Hygenic Corporation, USA). The training program was designed to be progressive using an undulating design with sets ranging between 2-4 and training intensity ranging between $12-20$ repetitions depending on week of training. The training equipment used was light weight to allow for easy transportation when participants traveled between Air Force Bases. Participants received a training bag including: a head harness (Neck Flex, Gonzo Companies, USA), six color-coded levels of resistance bands (red, green, blue, black, silver and gold), exercise handles, and a door anchor (Thera-Band ${ }^{\circ}$, The Hygenic Corporation, USA), and a training manual that described all training exercises in detail. In addition, participants were given online access to a training homepage with supplementary training information and training videos for each exercise. All participants received a personal training diary that described when to perform the various training exercises. Training was to be performed within working hours or if preferred at leisure.

\section{Training adherence}

Training was based on self-management education due to a dynamic work schedule among participants and frequent travel between Air Force Bases. At the beginning of the intervention all participants received an individual or group introduction to the training program. The introduction included: 1) a detailed description of the training program and diary, 2) an introduction to all training exercises and adjustments to ensure high quality on exercise performance, and 3) practical information regarding supervision during the intervention period. Participants received at least one follow-up visit during the intervention period in order to make sure that training exercises were performed correctly, and with progression. To motivate participants in the ETG to train, motivational posters were hung on the walls in the rooms of the two squadrons and tweets were posted on 
the training homepage. Thus, the training was not regularly supervised but was self-administered with the above support actions.

\section{Statistical analysis}

A pre-intervention power analysis was performed on the single primary outcome variable of self-reported intensity of neck pain previous 3-months [13]. Pain intensity was rated on an 11 point numeric box scale. The analysis showed that we would need to include 54 participants (27 experimental subjects and 27 control subjects) in this study. The analysis was based on the finding that a change of 1 measured on a 11 point numeric box scale is considered the minimum clinically significant difference regarding change in pain [23]. We also used results on pain intensity from a previous study among military pilots that found the response within this subject group to be normally distributed with a standard deviation in neck pain intensity of 1.5 the previous 3-months [24]. With a power set at 0.8 and a probability of a type I error of $p<0.05$, we would be able to detect a true difference in mean response of neck pain between experimental and control subjects equal to \pm 1.2 measured on an 11 point numeric box scale. Allowing for a $10 \%$ loss to follow-up, the total number of participants required was 64. The null-hypothesis (no difference between experimental and control subjects) was to be rejected if a between-group-difference for intensity of neck pain previous 3 -months was significant $(p<0.05)$. The relationship between intensity of neck and shoulder pain previous 3-months, and pilots' and crew-members: age, height, sitting height, weight, BMI, flying hours in fixed-wing aircraft, flying hours in rotary-wing aircraft, and flying hours with NVG, was analysed by multiple regression. Two statistical analyses were performed: 1) an intention-to-treat analysis comparing participants in ETG and REF as originally allocated after randomization [25], and 2) a per-protocol analysis only including participants in ETG that adhered regularly to the exercise intervention. Regular training adherence was defined as training between 1-3 times a week during the 20-week intervention period ( $\geq 33.3 \%$ of the total amount of training sessions). For missing values last observation carried forward or backwards were imputed. If observations were missing at both baseline and follow-up, the population mean was imputed at baseline, and group mean (ETG or REF) at follow-up. Between-groupdifferences at baseline were analyzed using the Student's $t$ test. The same analysis was performed at follow-up, including an analysis on delta-values (calculated by subtracting the pre- from post-intervention values). Within-groupchanges were analyzed by a paired $t$-test. The level of statistical significance was $p<0.05$. Results are presented as sample means and standard deviations (mean $\pm \mathrm{SD}$ ) if not otherwise specified. Statistical analyses were performed in
Stata Statistics/Data Analysis version 13.0 (StataCorp LP, USA).

\section{Results \\ Pre-intervention \\ Participant characteristics}

Overall mean $( \pm \mathrm{SD})$ for all participants was for age: 40 years $(40.6 \pm 7.6$ years $)$, height: $1.81 \mathrm{~m}(1.81 \pm 0.1 \mathrm{~m})$, and weight: $84 \mathrm{~kg}(83.9 \pm 12.2 \mathrm{~kg})$. No significant preintervention differences pertaining to anthropometric values or flight experience were identified between the ETG and REF (Table 1).

\section{Pain prevalence}

The 12-month prevalence of neck pain was $90.3 \%$ for helicopter pilots. Of these $54.8 \%$ had experienced 1-7 days with neck pain, $32.3 \%$ had experienced 8-30 pain days, and $3.2 \%$ had experienced $>90$ days with neck pain. For crew-members the 12-month prevalence of neck pain was $81.6 \%$. Of these, $44.7 \%$ had experienced 1-7 pain days, $29.0 \%$ had experienced $8-30$ pain days, $2.6 \%$ had experienced $>90$ pain days, and $5.3 \%$ had experienced neck pain daily. The 12-month prevalence of shoulder pain (right/left side) was 54.8\% / 32.3\% for pilots, and $42.1 \%$ / $39.5 \%$ for crew. No significant preintervention differences were found between the ETG and REF group regarding pain intensity, neither in the neck nor the shoulder within the previous 3 -months or 7-days (Table 2).

\section{Pain intensity and sensitivity}

Self-reported pre-intervention intensity of neck pain previous 3-months for the whole group was: $2.2 \pm 1.8$. Preintervention pain sensitivity as assessed by PPT in TRA and UNE (mean of right and left) was: $429 \pm 182 \mathrm{kPa}$ and $358 \pm 160 \mathrm{kPa}$ in ETG, and $411 \pm 166 \mathrm{kPa}$ and $334 \pm 151$ $\mathrm{kPa}$ in REF. No significant between-group-differences were

Table 1 Participants' characteristics

\begin{tabular}{lccc}
\hline & ETG $(n=35)$ & REF $(n=34)$ & $p$-value \\
\hline Age (years) & $40.4 \pm 6.7$ & $40.7 \pm 8.4$ & 0.880 \\
Height $(\mathrm{m})$ & $1.82 \pm 0.07$ & $1.80 \pm 0.08$ & 0.360 \\
Seated height $(\mathrm{m})$ & $0.95 \pm 0.05$ & $0.95 \pm 0.04$ & 0.995 \\
Weight (kg) & $84.2 \pm 12.7$ & $83.7 \pm 11.8$ & 0.882 \\
$\begin{array}{l}\text { Body mass index } \\
\text { (BMI) }\end{array}$ & $25.4 \pm 3.0$ & $25.7 \pm 2.3$ & 0.640 \\
$\begin{array}{l}\text { Flying hours in fixed-wing } \\
\text { aircraft (hours) }\end{array}$ & $226.3 \pm 565.3$ & $124.1 \pm 371.5$ & 0.523 \\
$\begin{array}{l}\text { Flying hours in rotary-wing } \\
\text { aircraft (hours) }\end{array}$ & $1778.1 \pm 1214.3$ & $2142.8 \pm 1451.1$ & 0.374 \\
$\begin{array}{l}\text { Flying hours with } \\
\text { NVG (hours) }\end{array}$ & $160.6 \pm 99.1$ & $174.6 \pm 142.0$ & 0.995 \\
\hline
\end{tabular}

Values are presented as mean \pm SD. Exercise-training-group (ETG), Reference-group (REF), Night vision goggles (NVG) 
Table 2 Intention-to-treat analysis of neck and shoulder pain intensity the previous 3-months and 7-days

\begin{tabular}{|c|c|c|c|c|}
\hline & $\mathrm{ETG}(n=35)$ & $\operatorname{REF}(n=34)$ & (95\% Conf. Interval) & $p$-value \\
\hline \multicolumn{5}{|c|}{ Neck pain previous 3-months } \\
\hline Pre-intervention & $1.9 \pm 1.7$ & $2.5 \pm 1.9$ & $-0.6(-1.5-0.3)$ & 0.159 \\
\hline Post-intervention & $1.9 \pm 2.0$ & $1.9 \pm 1.8$ & $-0.1(-1.0-0.8)$ & 0.865 \\
\hline Change & $0.0 \pm 2.5$ & $-0.5 \pm 1.7$ & $0.5(-0.5-1.6)$ & 0.291 \\
\hline \multicolumn{5}{|c|}{ Neck pain previous 7-days } \\
\hline Pre-intervention & $1.0 \pm 1.4$ & $1.0 \pm 1.5$ & $0.1(-0.7-0.8)$ & 0.871 \\
\hline Post-intervention & $0.6 \pm 1.1$ & $0.7 \pm 1.4$ & $-0.1(-0.7-0.5)$ & 0.724 \\
\hline Change & $-0.5 \pm 1.1 \dagger$ & $-0.3 \pm 1.4$ & $-0.2(-0.8-0.5)$ & 0.602 \\
\hline \multicolumn{5}{|c|}{$\begin{array}{l}\text { Shoulder pain (right side) } \\
\text { previous 3-months }\end{array}$} \\
\hline Pre-intervention & $1.3 \pm 2.0$ & $1.3 \pm 1.5$ & $0.0(-0.8-0.9)$ & 0.951 \\
\hline Post-intervention & $1.1 \pm 1.8$ & $1.6 \pm 2.2$ & $-0.5(-1.4-0.5)$ & 0.327 \\
\hline Change & $-0.1 \pm 2.1$ & $0.4 \pm 1.6$ & $-0.5(-1.4-0.4)$ & 0.267 \\
\hline \multicolumn{5}{|c|}{$\begin{array}{l}\text { Shoulder pain (right side) } \\
\text { previous 7-days }\end{array}$} \\
\hline Pre-intervention & $0.7 \pm 1.7$ & $0.5 \pm 1.1$ & $0.2(-0.5-0.9)$ & 0.529 \\
\hline Post-intervention & $0.6 \pm 1.6$ & $0.9 \pm 1.9$ & $-0.3(-1.1-0.6)$ & 0.515 \\
\hline Change & $-0.1 \pm 1.9$ & $0.3 \pm 1.2$ & $-0.5(-1.3-0.3)$ & 0.213 \\
\hline \multicolumn{5}{|c|}{$\begin{array}{l}\text { Shoulder pain (left side) } \\
\text { previous 3-months }\end{array}$} \\
\hline Pre-intervention & $0.8 \pm 1.5$ & $0.9 \pm 1.5$ & $-0.0(-0.8-0.7)$ & 0.913 \\
\hline Post-intervention & $0.7 \pm 1.3$ & $0.6 \pm 1.2$ & $0.0(-0.6-0.6)$ & 0.969 \\
\hline Change & $-0.2 \pm 1.6$ & $-0.2 \pm 1.6$ & $0.1(-0.7-0.8)$ & 0.895 \\
\hline \multicolumn{5}{|c|}{$\begin{array}{l}\text { Shoulder pain (left side) } \\
\text { previous 7-days }\end{array}$} \\
\hline Pre-intervention & $0.4 \pm 1.1$ & $0.5 \pm 1.3$ & $-0.1(-0.7-0.5)$ & 0.694 \\
\hline Post-intervention & $0.4 \pm 1.0$ & $0.0 \pm 0.2$ & $0.3(0.0-0.7)$ & $0.049^{*}$ \\
\hline Change & $-0.1 \pm 1.3$ & $-0.5 \pm 1.3 \dagger$ & $0.5(-0.2-1.1)$ & 0.149 \\
\hline
\end{tabular}

Values are presented as mean \pm SD. Significant between-group-difference is denoted by *. Significant within-group-change is denoted by + . Exercise-traininggroup (ETG), Reference-group (REF)

found regarding pain intensity or PPT measurements preintervention (Tables 2 and 3).

\section{Associated pain factors}

Among pilots, the intensity of left shoulder pain previous 3 -months was significantly related to age $(p=0.040)$ and flying hours in a fixed wing aircraft $(p=0.005)$. Intensity of neck pain previous 3-months among crew-members was found significantly related to seated height $(p=0.007)$. No significant relation was found between pain intensity, height, weight, BMI, flying hours in a helicopter, or flying hours with NVG for neither pilots nor crew-members.

\section{Post-intervention}

Pain intensity

Regarding the primary outcome of intensity of neck pain previous 3-months, no significant between-groupdifference or between-group-change, was found post- intervention (Table 2). Only, with regards to the secondary outcome, intensity of left shoulder pain previous 7-days, a between-group-difference was significant. Additionally, a significant within-group-change was found in ETG regarding intensity of neck pain previous 7-days (from: $1.0 \pm 1.4$, to: $0.6 \pm 1.1$, change: $-0.5 \pm 1.1 \quad(p=0.024))$ (Table 2).

\section{Pain sensitivity, PPT}

Regarding the secondary outcome of PPT no significant between-group-differences or between group change were found post-intervention (Table 3). However, significant within-group-changes were observed for the ETG regarding PPT in the left TRA (change: $-53 \pm 113 \mathrm{kPa}, p=0.009)$ and for the reference point (change: $-56 \pm 125 \mathrm{kPa}, p=0.012$ ). For participants in REF a significant-within-group change was found for PPT in the TRA right (change: $-74 \pm 137 \mathrm{kPa}, p=$ 
Table 3 Intention-to-treat analysis for pressure-pain-threshold in neck and shoulder muscles

\begin{tabular}{|c|c|c|c|c|}
\hline & $\mathrm{ETG}(n=35)$ & $\operatorname{REF}(n=34)$ & (95\% Conf. Interval) & $p$-value \\
\hline \multicolumn{5}{|l|}{ Trapezius m. (right) } \\
\hline Pre-intervention (kPa) & $424 \pm 187$ & $416 \pm 177$ & $8(-79-96)$ & 0.851 \\
\hline Post-intervention (kPa) & $409 \pm 183$ & $342 \pm 143$ & $67(-12-146)$ & 0.094 \\
\hline Change (kPa) & $-15 \pm 126$ & $-74 \pm 137 \dagger$ & $59(-4-122)$ & 0.067 \\
\hline \multicolumn{5}{|l|}{ Trapezius m. (left) } \\
\hline Pre-intervention (kPa) & $434 \pm 188$ & $405 \pm 163$ & $28(-56-113)$ & 0.507 \\
\hline Post-intervention (kPa) & $381 \pm 169$ & $332 \pm 154$ & $49(-29-127)$ & 0.211 \\
\hline Change (kPa) & $-53 \pm 113+$ & $-74 \pm 119+$ & $21(-35-77)$ & 0.456 \\
\hline \multicolumn{5}{|c|}{ Upper neck extensors (right) } \\
\hline Pre-intervention (kPa) & $345 \pm 157$ & $334 \pm 147$ & $11(-62-84)$ & 0.759 \\
\hline Post-intervention (kPa) & $347 \pm 152$ & $295 \pm 116$ & $53(-12-118)$ & 0.110 \\
\hline Change (kPa) & $2 \pm 118$ & $-39 \pm 97 \dagger$ & $41(-11-93)$ & 0.117 \\
\hline \multicolumn{5}{|c|}{ Upper neck extensors (left) } \\
\hline Pre-intervention (kPa) & $371 \pm 170$ & $335 \pm 163$ & $36(-44-116)$ & 0.377 \\
\hline Post-intervention (kPa) & $348 \pm 146$ & $292 \pm 121$ & $56(-8-121)$ & 0.086 \\
\hline Change (kPa) & $-23 \pm 110$ & $-44 \pm 104 \dagger$ & $21(-31-72)$ & 0.424 \\
\hline \multicolumn{5}{|l|}{ Reference point } \\
\hline Pre-intervention (kPa) & $643 \pm 218$ & $622 \pm 231$ & $21(-87-129)$ & 0.700 \\
\hline Post-intervention (kPa) & $587 \pm 209$ & $553 \pm 224$ & $34(-70-139)$ & 0.513 \\
\hline Change (kPa) & $-56 \pm 125 \dagger$ & $-69 \pm 209$ & $13(-69-96)$ & 0.746 \\
\hline
\end{tabular}

Values are presented as mean \pm SD. Significant within-group-change is denoted by †. Exercise-training-group (ETG), Reference-group (REF)

0.003 ), and left (change: $-74 \pm 119 \mathrm{kPa}, p=0.001$ ), and for the UNE right (change: $-39 \pm 97 \mathrm{kPa}, p=$ 0.024 ) and left (change: $-44 \pm 104 \mathrm{kPa}, p=0.045$ ). Overall, REF increased pain sensitivity in more sites in the neck and shoulder than the ETG.

\section{Training adherence}

Twenty-five participants out of 35 (71.4\%) within the ETG returned the post-intervention questionnaire regarding training adherence. Of these, 10 participants $(\sim 30 \%)$ reported adhering to training regularly between 1-3 times a week throughout the intervention period (Fig. 2). The per-protocol-analysis including participants that adhered regularly to training $(n=10)$ demonstrated no additional significant between-group-differences regarding the primary outcome of intensity of neck pain previous 3-months. Similarly, regarding the secondary outcome values of PPT and additional neck/shoulder pain intensity variables no significant differences were found. However, an additional within-group-change was significant in ETG regarding intensity of neck pain previous 3 -months (from: $2.2 \pm 0.6$, to: $1.3 \pm 1.3$, change: $-0.9 \pm 1.0(p=0.019))$ (Fig. 3). Further, intensity of neck pain previous 7-days decreased significantly (from: $1.4 \pm$ 0.8 , to: $0.5 \pm 0.8$, change: $-0.9 \pm 1.1(p=0.029))$, in line with results from the intention-to-treat analysis (Table 2 ).

\section{Discussion}

The main finding of this randomized controlled trial was that 20-weeks of strength, endurance, and coordination training, targeting the neck and shoulder muscles, provided no significant effect on intensity of neck pain previous 3-months, i.e. our primary hypothesis was rejected. However, of major interest was the finding of a high prevalence of neck pain among helicopter pilots and

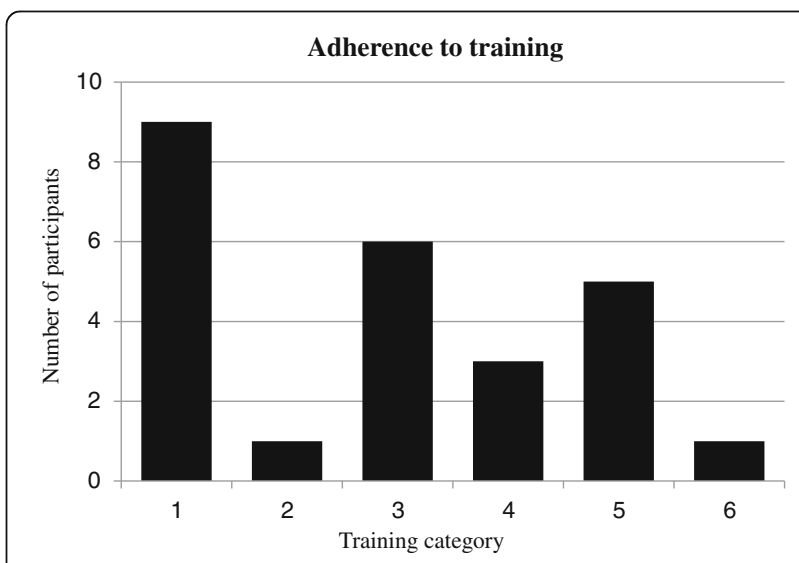

Fig. 2 Training adherence; 1) trained regularly 2 - 3 times a week, 2) trained regularly $1-2$ times a week, 3) trained irregular, but at least 4 times a month (approximately once a week), 4) trained irregularly but at least $2-3$ times a month, 5) trained some, but stopped training after a while, 6) did not use the training offer 


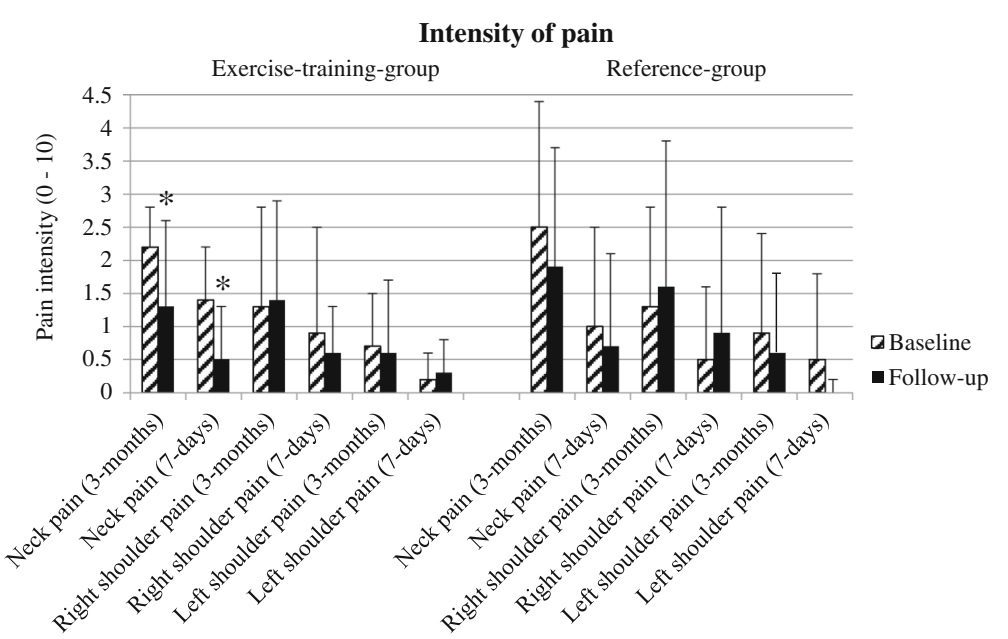

Fig. 3 Intensity of neck and shoulder pain analyzed by per-protocol analysis (ETG, $n=10$ and REF, $n=34$ ). Values are presented as mean $\pm S D$. Significant within-group-changes are denoted by*

crew-members. Furthermore, a significant secondary outcome regarding a within-group-effect of reduced pain intensity and sensitivity, particularly in ETG, was found.

\section{Pain intensity}

The primary outcome of intensity of neck pain previous 3months was lower than expected compared with previous studies. Ang et al. previously reported the 3-months intensity of neck pain among helicopter pilots equal to 4.4 using the Borg Category-Ratio scale (CR 0-10 scale) [2]. The scale used by Ang et al. is equal to the one used in our study and results are therefore comparable. Intensity of pain was higher in the study by Ang et al. as compared to our results. Participants within our study constituted the majority of helicopter pilots and crew-members within the RDAF and are therefore considered representative in relation to other helicopter pilots and crew-members. In the study by Ang et al. only pilots who reported pain as "once or a few times over the previous 3-months" were included in the analysis [2]. In comparison, no pain threshold was used to identify pilots or crew-members in our study. This was a deliberate decision to assess if the exercise intervention would be useful in relation to pain prevention. Undoubtedly, this decision has influence on the average intensity of pain among participants. Notably, a decrease in pain is less likely when a low baseline pain level is present due to the "diluting" effect of participants with no pain.

\section{Pain sensitivity, PPT}

Regarding the secondary outcome of PPT the exercise intervention provided no significant between-groupdifferences. However, a significant reduction in PPT was observed in REF in both TRA and UNE from baseline to follow-up. In comparison, PPT was only significantly reduced in left TRA in ETG. It should be emphasized, that a reduction in PPT is equal to an increase in pain sensitivity. Based on our results, the exercise intervention may therefore have had some impact on pain sensitivity in the neck and shoulder muscles. The exercise intervention was planned during the winter period that includes many flight hours with NVG due to low light conditions. Based on previous studies, demonstrating the impact of head-worn mass by NVG, the winter months must be regarded as the most stressful to pilots and crew-members with respect to cervical load. The exercise intervention may therefore have provided some form of pain relief as pain sensitivity in the neck and shoulder muscles among participants in ETG did not undergo the same significant increase as observed in REF. This needs further investigation as no significant between-group-differences were present. To our knowledge, no previous studies have measured PPT in neck and shoulder muscles among helicopter pilots and crew. However, exercise has been shown to increase pressure-pain-threshold in neck muscles in response to short [26, 27] and longer training interventions [28]. Pressure pain threshold may be considered a "semi-objective" measurement of pain sensitivity because participants mark, in a blinded setting, the incidence of pain occurrence, while increasing pressure is being applied, resulting in the reading of the actual numerical outcome of pressure when the threshold is met [29]. The participants may have underreported the sensation of pain because pain complaints may be regarded as a sign of weakness among military pilots [24]. This would certainly impact the study's potential regarding additional increases in PPT.

\section{Pain prevalence and duration}

Neck pain was highly prevalent among participants with a 1-year prevalence amounting to $90.3 \%$ for pilots and $81.6 \%$ for crew-members, respectively. Our results correspond 
with those of other studies surveying the 1-year prevalence of neck pain among helicopter pilots and crew-members. Van den Oord et al. previously reported a 1-year prevalence equal to $43 \%$ for Dutch military helicopter pilots [30] and $62 \%$ for crew-members [5]. In comparison, Birger et al. reported a 1-year prevalence of $48 \%$ among British helicopter pilots [31]. Our prevalence rates are higher compared to these previous studies. However, discrepancies may be explained by use of different pain definitions and pain cut points. Van den Oord et al. defined pain as "any pain, ache or discomfort" using four pain categories (never, occasional, regular, or continuous) $[5,30]$. In the study by Birger et al, no distinctions were made between occasional, regular, or continuous neck pain [31]. In comparison, we also defined pain as "any pain" within our study but used six pain categories (0 days, $1-7$ days, $8-30$ days, $31-90$ days, $>90$ days, every day). The use of additional pain categories may attribute to the higher 1-year prevalence for any pain among pilots and crew within our study. Based on fairly homogeneous neck pain definitions, the 1-year prevalence within the general adult population (17 - 70 years of age) has been reported to range between $17 \%-75 \%$ with a mean of $37 \%$ [3]. The prevalence of neck pain among helicopter pilots and crew-members must therefore be regarded as high with potential impact on individual health and overall well-being [3] and interference with flying [2]. Neck-pain among helicopter pilots and crew has previously been described as chronic [1]. Our data, however, do not support this definition as the majority of pilots $(87.1 \%)$ and crew-members (73.7\%) in our study reported pain durations of between one and thirty days within the last year. In comparison, only $3.2 \%$ of pilots and $2.6 \%$ of crew-members reported more than 90 pain days. Adhering to the definition of "chronic pain", the sensation of pain must be persistent with a duration of three months or more a year [32]. Based on our findings, neck pain among this occupational group may be more appropriately described as "episodic neck pain". Interestingly, pilots and crew-members have been found reluctant to report pain due to fear of flying restrictions [33] and jeopardize future employment opportunities or pension entitlements [2]. Lastly, pilots and crew fulfilling the highest demands of health in the RDAF will report a lower intensity of pain, as opposed to studies conducted on patient groups seeking medical treatment for pain symptoms [34]. Future studies may obtain valuable knowledge by considering measuring the influence of neck pain, in comparison to pain intensity itself, within this specific occupational group.

\section{Training adherence}

Adherence to training is a challenge and affects the results of an intervention. The lack of a clear intervention effect on pain intensity and PPT may therefore have been influenced by adherence, as only $\sim 1 / 3$ of participants in ETG trained regularly. Previously, self-reported adherence to training and actual training participation has been found reliable $[35,36]$. However, adherence was analyzed according to $71.4 \%$ of questionnaire respondents, leaving some uncertainty regarding transferability to the remaining $28.6 \%$ in ETG. In a previously conducted randomized controlled trial aiming to reduce neck pain among Swedish helicopter pilots, $77 \%$ of participants adhered to the prescribed daily regime during a 6-week intervention period [37]. In addition, in a former 12-week exercise study on helicopter aircrew within the Canadian forces, aiming to improve neck muscle function, regular training adherence was achieved in $52.8 \%$ of participants randomized to neck endurance training, and in $76.1 \%$ of participants randomized to neck coordination training [38]. Finally, a recently conducted exercise intervention, aiming to reduce neck and shoulder pain among Danish fighter pilots, 58\% of participants adhered to training 1-3 times a week throughout a 24-week intervention period [24]. When compared to our results $(28.6 \%)$, adherence within our study must be regarded as low. Exercise training must be performed regularly to prove beneficial and the low level of adherence within our study may not be sufficient to conclude on the full effectiveness of the exercise intervention itself. Previously, the importance of supervised training to enhance compliance among aircrew has been underlined [39]. However, uncertainty still exists regarding the benefit of supervised training in general. As demonstrated by Gram et al., 20-weeks of physical exercise training at the workplace (one hour per week) may be highly effective in reducing neck pain, independent of the level of supervision [40]. Due to logistical reasons, supervision was not optional within our study. Pilots and crew have very dynamic work schedules and work on different air bases placed throughout the country, and we therefore designed the intervention to be self-administered. Future studies may have to put effort on how pilots and crew may be followed up frequently, if adherence to training is to be increased, e.g. by the use of information and communication technologies.

\section{Limitations}

Some limitations of the present study must be considered when interpreting data and results. The low level of regular training adherence within ETG must be regarded as a primary limitation. Low adherence reduces the statistical power of the study, and may therefore also have undermined the intervention and increased the risk of erroneous conclusions and consequently a false rejection of an effective exercise intervention. Also, pilots and crew members who had left their flying duties or the military, perhaps because of severe neck pain problems, are not included in this study. The study included subjects with and without neck pain to investigate if specific neck training could reduce the incidence of flight related 
neck pain. However, including subjects without pain at baseline will impact on the possible overall level of pain within groups, and also the potential to reduce pain. It may be argued that a measurement of flight-related pain would have proven better than a questionnaire assessing intensity of pain previous 3-months and 7-days. Flight related neck pain has been found to develop during and after sorties, and typically last for a day or two. Therefore, it would have been interesting to measure intensity of pain the day after each sortie within the intervention period in order to provide a better understanding of the pain patterns in relation to type of sortie. However, this was not performed in the present study.

\section{Conclusions}

The exercise intervention demonstrated some preventive properties as some neck pain intensity and sensitivity improved within ETG but not within REF. However, no significant between-group-differences were present. The lack of a clear intervention effect on pain intensity may be due to low adherence as only $\sim 1 / 3$ of subjects in the ETG engaged in regular training, which may be due to the self-administration of the training regime.

\section{Abbreviations}

BMI: Body mass index; ETG: Exercise-training-group; NVG: Night vision goggles; PPT: Pressure-pain-threshold; RDAF: Royal Danish Air Force; REF: Reference-group; SD: Standard deviation; TIA: Anterior tibialis muscle; TRA: Trapezius muscle; UNE: Upper neck muscles

\section{Acknowledgements}

The authors would like to thank the Royal Danish Air Force for financial funding. We would also like to express our gratitude to Phil Page, Global Director of Clinical Education and Research at the Hygenic Corporation, for the donation of free Thera-Band products for the present study.

\section{Funding}

This study was financially funded by the Royal Danish Air Force and the University of Southern Denmark.

\section{Availability of data and materials}

The datasets generated and analysed during the current study are not publicly available but may be accessible from the authors upon reasonable request and permission from The Royal Danish Airforce.

\section{Authors' contributions}

The authors MM, GS, KS, BL, and BRN have made substantial contribution to the conception and design. MM performed the experimental testing and implementation of the intervention as well as data acquisition. The authors MM, GS, and KS were involved in data analysis and additionally BL and BRN were involved in interpretation, writing, and critically revising the paper for important intellectual content. All authors have given final approval of the version to be published and agreed to be accountable for all aspects of the work.

\section{Competing interests}

The authors declare that they have no competing interests.

\section{Consent for publication}

Not applicable.

\section{Ethics approval and consent to participate}

The trial was approved by the local Ethics Committee of Southern Denmark (S-20120121) and qualified for registration in ClinicalTrials.gov (NCT01926262).

\section{Publisher's Note}

Springer Nature remains neutral with regard to jurisdictional claims in published maps and institutional affiliations.

\section{Author details}

'Department of Sports Science and Clinical Biomechanics, University of Southern Denmark, Odense, Denmark. ${ }^{2}$ Department of Anesthesia and Intensive Care Medicine, Odense University Hospital, Odense, Denmark. ${ }^{3}$ Royal Danish Air Force, Air Force Staff, Defence Command Denmark, Karup, Denmark.

Received: 28 December 2016 Accepted: 29 March 2017

Published online: 07 April 2017

\section{References}

1. Salmon DM, Harrison MF, Neary JP. Neck pain in military helicopter aircrew and the role of exercise therapy. Aviat Space Environ Med. 2011;82(10):978-87.

2. Ang B, Harms-Ringdahl K. Neck pain and related disability in helicopter pilots: a survey of prevalence and risk factors. Aviat Space Environ Med. 2006;77(7):713-9.

3. Fejer R, Kyvik KO, Hartvigsen J. The prevalence of neck pain in the world population: a systematic critical review of the literature. Eur Spine J. 2006; 15(6):834-48.

4. Adam J. Results of NVG-induced neck strain questionnaire study in $\mathrm{CH}-146$ Griffon aircrew. In: Defence R\&D Canada. Toronto: DRDC Toronto Technical Report No. 2004-153; 2004.

5. Van den Oord MHA, Sluiter JK, Frings-Dresen MHW. Differences in physical workload between military helicopter pilots and cabin crew. Int Arch Occup Environ Health. 2014;87(4):381-6.

6. Harrison MF, Coffey B, Albert WJ, Fischer SL. Night vision goggle-induced neck pain in military helicopter aircrew: a literature review. Aviat Space Environ Med. 2015;86(1):46-55.

7. Thuresson M, Ang B, Linder J, Harms-Ringdahl K. Neck muscle activity in helicopter pilots: effect of position and helmet-mounted equipment. Aviat Space Environ Med. 2003;74(5):527-32.

8. Thuresson M, Ang B, Linder J, Harms-Ringdahl K. Mechanical load and EMG activity in the neck induced by different head-worn equipment and neck postures. Int J Ind Ergon. 2005:35(1):13-8.

9. Murray M, Lange B, Chreiteh SS, Olsen HB, Nørnberg BR, Boyle E, Sogaard K, Sjogaard G. Neck and shoulder muscle activity and posture among helicopter pilots and crew-members during military helicopter flight. J Electromyogr Kinesiol. 2016;27(2):10-7.

10. Zebis MK, Andersen LL, Pedersen MT, Mortensen P, Andersen $\mathrm{CH}$, Pedersen MM, Boysen M, Roessler KK, Hannerz H, Mortensen OS, et al. Implementation of neck/shoulder exercises for pain relief among industrial workers: a randomized controlled trial. BMC Musculoskelet Disord. 2011;12:205.

11. Andersen LL, Andersen CH, Zebis MK, Nielsen PK, Sogaard K, Sjogaard G. Effect of physical training on function of chronically painful muscles: a randomized controlled trial. J Appl Physiol. 2008:105(6):1796-801.

12. Blangsted AK, Sogaard K, Hansen EA, Hannerz H, Sjogaard G. One-year randomized controlled trial with different physical-activity programs to reduce musculoskeletal symptoms in the neck and shoulders among office workers. Scand J Work Environ Health. 2008;34(1):55-65.

13. Murray M, Lange B, Nornberg BR, Sogaard K, Sjogaard G. Specific exercise training for reducing neck and shoulder pain among military helicopter pilots and crew members: a randomized controlled trial protocol. BMC Musculoskelet Disord. 2015;16:198.

14. Rasotto C, Bergamin M, Simonetti A, Maso S, Bartolucci GB, Ermolao A, Zaccaria M. Tailored exercise program reduces symptoms of upper limb work-related musculoskeletal disorders in a group of metalworkers: a randomized controlled trial. Man Ther. 2015;20(1):56-62.

15. Sjogaard G, Justesen JB, Murray M, Dalager T, Sogaard K. A conceptual model for worksite intelligent physical exercise training - IPET - intervention for decreasing life style health risk indicators among employees: a randomized controlled trial. BMC Public Health. 2014;14:652.

16. Holtermann A, Roeleveld K, Mork PJ, Gronlund C, Karlsson JS, Andersen LL, Olsen HB, Zebis MK, Sjogaard G, Sogaard K. Selective activation of neuromuscular compartments within the human trapezius muscle. J Electromyogr Kinesiol. 2009;19(5):896-902.

17. Juul-Kristensen B, Laursen B, Pilegaard M, Jensen BR. Physical workload during use of speech recognition and traditional computer input devices. Ergonomics. 2004;47(2):119-33. 
18. Gosselin G, Rassoulian H, Brown I. Effects of neck extensor muscles fatigue on balance. Clin Biomech. 2004;19(5):473-9.

19. Rosendal L, Larsson B, Kristiansen J, Peolsson M, Sogaard K, Kjaer M, Sorensen J, Gerdle B. Increase in muscle nociceptive substances and anaerobic metabolism in patients with trapezius myalgia: microdialysis in rest and during exercise. Pain. 2004;112(3):324-34.

20. Persson AL, Hansson GA, Kalliomaki J, Moritz U, Sjolund BH. Pressure pain thresholds and electromyographically defined muscular fatigue induced by a muscular endurance test in normal women. Clin J Pain. 2000;16(2):155-63.

21. Kuorinka I, Jonsson B, Kilbom A, Vinterberg H, Bieringsorensen F, Andersson G, Jorgensen K. Standardized nordic questionnaires for the analysis of musculoskeletal symptoms. Appl Ergon. 1987;18(3):233-7.

22. Specific exercise training for helicopter pilots and crew-members [www.sdu dk/helicopter]. Accessed 16 Aug 2016.

23. Kelly AM. Does the clinically significant difference in visual analog scale pain scores vary with gender, age, or cause of pain? Acad Emerg Med. 1998:5(11): 1086-90.

24. Lange B, Toft P, Myburgh C, Sjogaard G. Effect of targeted strength, endurance, and coordination exercise on neck and shoulder pain among fighter pilots a randomized-controlled trial. Clin J Pain. 2013;29(1):50-9.

25. Hollis S, Campbell F. What is meant by intention to treat analysis? Survey of published randomised controlled trials. Br Med J. 1999:319(7211):670.

26. Nielsen PK, Andersen LL, Olsen HB, Rosendal L, Sjogaard G, Sogaard K. Effect of physical training on pain sensitivity and trapezius muscle morphology. Muscle Nerve. 2010;41(6):836-44.

27. Andersen $L L$, Andersen $\mathrm{CH}$, Sundstrup $\mathrm{E}$, Jakobsen MD, Mortensen $\mathrm{OS}$, Zebis MK. Central adaptation of pain perception in response to rehabilitation of musculoskeletal pain: randomized controlled trial. Pain Physician. 2012;15(5):385-94.

28. Ylinen J, Takala EP, Kautiainen $H$, Nykanen M, Hakkinen A, Pohjolainen T, Karppi SL, Airaksinen O. Effect of long-term neck muscle training on pressure pain threshold: a randomized controlled trial. Eur J Pain. 2005; 9(6):673-81.

29. Andersen CH, Andersen LL, Zebis MK, Sjogaard G. Effect of scapular function training on chronic pain in the neck/shoulder region: a randomized controlled trial. J Occup Rehabil. 2014;24(2):316-24.

30. Van den Oord MHA, De Loose V, Meeuwsen T, Sluiter JK, Frings-Dresen MHW. Neck pain in military helicopter pilots: prevalence and associated factors. Milit Med. 2010;175(1):55-60.

31. Bridger RS, Groom MR, Jones H, Pethybridge RJ, Pullinger N. Task and postural factors are related to back pain in helicopter pilots. Aviat Space Environ Med. 2002;73(8):805-11.

32. Misailidou V, Malliou P, Beneka A, Karagiannidis A, Godolias G. Assessment of patients with neck pain: a review of definitions, selection criteria, and measurement tools. J Chiropr Med. 2010;9(2):49-59.

33. Harrison MF, Neary JP, Albert WJ, Veillette DW, McKenzie NP, Croll JC. Physiological effects of night vision goggle counterweights on neck musculature of military helicopter pilots. Milit Med. 2007:172(8):864-70.

34. Ylinen J. Physical exercises and functional rehabilitation for the management of chronic neck pain. Eura Medicophys. 2007:43(1):119-32.

35. Andersen $\mathrm{CH}$, Andersen LL, Pedersen MT, Mortensen P, Karstad K, Mortensen OS, Zebis MK, Sjogaard G. Dose-response of strengthening exercise for treatment of severe neck pain in women. J Strength Cond Res. 2013;27(12):3322-8.

36. Andersen LL, Jorgensen MB, Blangsted AK, Pedersen MT, Hansen EA, Sjogaard $G$. A randomized controlled intervention trial to relieve and prevent neck/ shoulder pain. Med Sci Sports Exerc. 2008:40(6):983-90

37. Ang BO, Monnier A, Harms-Ringdahl K. Neck/shoulder exercise for neck pain in air force helicopter pilots: a randomized controlled trial. Spine. 2009; 34(16):544-51.

38. Salmon DM, Harrison MF, Sharpe D, Candow D, Albert WJ, Neary JP. Exercise therapy for improved neck muscle function in helicopter aircrew. Aviat Space Environ Med. 2013;84(10):1046-54.

39. Alricsson M, Harms-Ringdahl K, Larsson B, Linder J, Werner S. Neck muscle strength and endurance in fighter pilots: effects of a supervised training program. Aviat Space Environ Med. 2004;75(1):23-8.

40. Gram B, Andersen C, Zebis MK, Bredahl T, Pedersen MT, Mortensen OS, Jensen $\mathrm{RH}$, Andersen $\mathrm{LL}$, Sjogaard $\mathrm{G}$. Effect of training supervision on effectiveness of strength training for reducing neck/shoulder pain and headache in office workers: cluster randomized controlled trial. Biomed Res Int. 2014:2014:9.

\section{Submit your next manuscript to BioMed Central and we will help you at every step:}

- We accept pre-submission inquiries

- Our selector tool helps you to find the most relevant journal

- We provide round the clock customer support

- Convenient online submission

- Thorough peer review

- Inclusion in PubMed and all major indexing services

- Maximum visibility for your research

Submit your manuscript at www.biomedcentral.com/submit
Biomed Central 